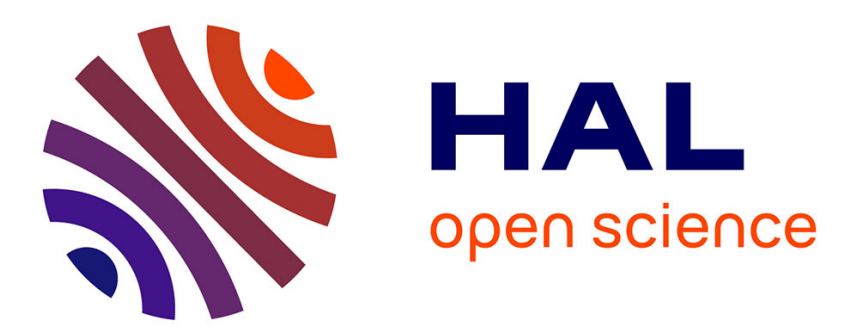

\title{
Post-transcriptional control of virulence gene expression in Staphylococcus aureus
}

\author{
Alexandre Le Scornet, Peter Redder
}

\section{To cite this version:}

Alexandre Le Scornet, Peter Redder. Post-transcriptional control of virulence gene expression in Staphylococcus aureus. Biochimica et Biophysica Acta - Gene Regulatory Mechanisms , 2019, 1862, pp.734 - 741. 10.1016/j.bbagrm.2018.04.004 . hal-03484705

\section{HAL Id: hal-03484705 https://hal.science/hal-03484705}

Submitted on 20 Dec 2021

HAL is a multi-disciplinary open access archive for the deposit and dissemination of scientific research documents, whether they are published or not. The documents may come from teaching and research institutions in France or abroad, or from public or private research centers.
L'archive ouverte pluridisciplinaire HAL, est destinée au dépôt et à la diffusion de documents scientifiques de niveau recherche, publiés ou non, émanant des établissements d'enseignement et de recherche français ou étrangers, des laboratoires publics ou privés.

\section{다)(1) $(5$}

Distributed under a Creative Commons Attribution - NonCommercial| 4.0 International 


\section{Post-transcriptional control of virulence}

\section{2 gene expression in Staphylococcus}

\section{3 aureus}

4

5 Authors:

6 Alexandre Le Scornet and Peter Redder

7 LMGM, Centre de Biologie Integrative,

8 Paul Sabatier University

9 118, Route de Narbonne

1031062 Toulouse.

11 France

12

13 Corresponding author: Peter Redder, peter.redder@univ-tlse3.fr

14

15

16 


\section{Abstract}

2 Opportunistic pathogens have to be ready to change life-style whenever the occasion arises, 3 and therefore need to keep tight control over the expression of their virulence factors. Doubly

4 so for commensal bacteria, such as Staphylococcus aureus, which should avoid harming 5 their hosts when they are in a state of peaceful co-existence. $S$. aureus carries very few 6 sigma factors to help define the transcriptional programs, but instead uses a plethora of 7 small RNA molecules and RNA-RNA interactions to regulate gene expression post8 transcriptionally. The endoribonucleases RNase III and RNase $\mathrm{Y}$ contribute to this regulatory 9 diversity, and provide a link to RNA-decay and intra-cellular spatiotemporal control of expression. In this review we describe some of these post-transcriptional mechanisms as well as some of the novel transcriptomic approaches that have been used to find and to study them.

\section{Introduction}

One of the major discoveries in the field of bacterial gene regulation, was that RNA molecules not only serve as passive intermediates between DNA and protein, but themselves play an important and active role in regulating both translation and transcription [1]. This realisation has led to the search, in recent years often by transcriptomic methods, for RNAs (or sub-sections of RNAs) with potential regulatory function. A number of excellent recent reviews dealt with the RNA-mediated regulatory mechanisms discovered in various bacteria and have outlined some of the methods used to probe/analyse the molecular details of these regulatory events [2-6].

In this review we highlight some particularly interesting examples of post-transcriptional regulation of virulence factors in Staphylococcus aureus, as well as describe some of the transcriptomic methods employed to gain a better understanding of these regulatory mechanisms, which until recently have been largely overlooked.

$S$. aureus is an opportunistic pathogen which benignly colonises the nasopharynx in $20-30 \%$ of the human population [7]. Under conditions such as weakened host immune system or direct access to the bloodstream, these same peaceful strains can change to aggressive pathogens, and need a highly organised gene regulatory system to ensure correct transition between the two lifestyles. In contrast to many bacteria, $S$. aureus does not depend on a large array of sigma factors to perform the fine-tuning of cellular programs, and only four sigma factors have been identified, two of them with cryptic expression conditions [8,9]. $S$. 
aureus relies instead on a finely tuned homeostasis between transcription regulators, small RNAs, RNA degradation, and intra-molecular RNA-RNA interactions to generate the required regulatory flexibility [2].

One of the most intriguing examples of RNA-mediated post-transcriptional regulation is RNAIll from S. aureus, which with its 14 hairpin structures can interact with, and regulate, a variety of RNA targets involved in virulence [2]. Bacterial mRNAs have short half-lives, typically below 3 minutes [10], and the steady-state level of a given RNA is therefore highly dependent on even minor changes in its degradation rate. What determines the degradation rate is unclear for most RNAs in $S$. aureus, but it appears that a "decay-initiating" endonucleolytic cleavage is the rate-limiting step, at least for a sub-set of RNAs. This is the case for about a hundred coding regions of RNA whose half-lives are dependent on the endonuclease RNase $Y$ (see also section 5) [11], as well as several cases in which the formation of an RNA-RNA duplex results in cleavage by the double-stranded RNase III (see also section 4) [2]. However, endonucleolytic cleavage can also be used to differentially regulate genes that are part of the same transcript, by dividing the transcript into two RNA molecules with different decay-rates $[12,13]$.

\section{The 5' and 3' untranslated regions}

Understanding the molecular details of post-transcriptional regulation, be it via secondary structures or degradation, requires detailed knowledge of the true extent of the RNA molecule examined. Most standard RNA sequencing protocols systematically lose sequence information from the ends of the RNAs. An example is the dUTP-based library preparation for RNA sequencing with Illumina technology (such as TruSeq), where the true 3'-end of the RNA is lost through use of random primers to generate the CDNA, and 5'-end information is lost when RNA fragments generated by RNase $\mathrm{H}$ treatment of the RNA-cDNA duplex are used as primers for generating the second DNA strand. However, in the last couple of years a range of novel methods have been developed which enable exact mapping of the RNA ends [14-20].

\subsection{Mapping the 5' ends}

Mapping of the 5'-ends have been the focus of most transcriptomic analyses, since this will not only reveal any potential hairpins that can sequester the ribosome binding site, or binding sites for small RNAs (see section 3, below), but also pinpoint the transcription start 
1 site on the genome (Figure 1). The most commonly used transcriptome-wide 5'-end 2 analyses involve ligating a synthetic RNA oligo to the 5' ends of all RNA in the sample 3 (Figure 1B), followed by high throughput RNA sequencing primed from the synthetic oligo 4 into the sequence of the cellular RNA. In silico analysis of the sequencing reads can find the known sequence of the oligo, and the 5'-end of the original RNA can then easily be identified downstream of the oligo sequence (Figure 1G).

7 A key question which often arises when analysing 5' ends, is whether a detected 5'-end is 8 tri-phosphorylated. Tri-phosphorylated nucleosides are present only at the original 5'-ends of 9 transcripts, and therefore indicate bona fide transcription start events (Figure 1A). Once the transcription start site is known, the full extent of the 5'-UTR is revealed, along with putative secondary structures and binding sites for small regulatory RNAs. In addition, once the transcription start site is mapped onto the genome of the organism, the pre-transcriptional regulation signals on the DNA, such as promoters and repressor binding sites, can often be 14 identified.

However, it is not trivial to determine whether a detected 5'-end is tri-phosphorylated, since RNA sequencing does not directly reveal the phosphorylation state of the transcript ends. In addition, ligation of an oligo to the 5'-ends (described above; see Figure 1B) can only be performed when the 5'-ends are mono-phosphorylated. Hence, it is necessary treat the RNA enzymatically, with a pyrophosphatase, to convert tri-phosphorylated 5'-ends to monophosphates (Figure 1E), thus losing any information about the original phosphorylation state! Note that this problem also besets non-transcriptomic 5'-end mapping methods such as primer extension or 5'-RACE, although the addition of a pyrophosphatase in the latter can help distinguish processed (i.e. mono-phosphorylated) from tri-phosphosphorylated 5'-ends [21-23].

The solution to this problem was the development of differential RNA sequencing (dRNAseq), in which RNA samples are split into two aliquots, each prepared separately for sequencing in a slightly different manner. One aliquot is digested with a 5' to 3' exoribonuclease, which is specific for mono-phosphorylated 5'-ends and will ignore the triphosphorylated RNA species. Both aliquots are then treated with a pyrophosphatase to convert tri-phosphates into mono-phosphates, followed by ligation to an oligo of known sequence. The two preparations are then converted to cDNA and deep-sequenced separately. Most of the transcriptome in the two samples will look highly similar, but careful comparison of the results from the two sequencings, either by inspection [24] or using sophisticated statistical methods [25-27] permits the identification of regions that are relatively enriched in the exoribonuclease-treated sample. Such an enrichment is caused by the exonuclease protection offered by the tri-phosphorylation, leading to a relative over- 
1 representation of the 5'-ends of tri-phosphorylated RNA fragments in the exoribonuclease-

2 treated aliquot [15].

\section{$4 \quad 2.2$ The 3'-ends, a relatively unexplored field}

5

6

7

Recently, it has been realised that the 3' UTRs of prokaryote RNA can also play regulatory roles. A global survey of 3' UTRs in S. aureus, using a combination of RNA sequencing, tiled micro-arrays and in silico analyses, found that 3' UTRs are generally much longer than they need to be to simply terminate transcription, suggesting that many 3' UTRs have additional functions [28]. This study furthermore showed that while rho-independent transcription terminators can be predicted in silico with reasonably good accuracy, they are often leaky and permit read-through, which further confound global analyses and structure predictions [28,29].

One function for the 3' UTR could be the formation of secondary structures to block 3' to 5' exoribonucleases, and thereby increase the half-life of the RNA, and this does indeed appear to be the case for some transcripts [30]. However, a highly intriguing mechanism was discovered for the $S$. aureus icaR transcript, which encodes the repressor of the operon for biosynthesis of exopolysaccharide for biofilm formation. The icaR transcript forms a giant hairpin with a duplex composed of the 5' UTR and the 3' UTR, which regulates IcaR translation on two levels. The first is the RNA half-life, which is lowered by the presence of the hairpin, because it serves as a substrate for the double stranded endoribonuclease RNase III (see section 4 below). This in turn leads to lowered levels of icaR RNA and of the encoded protein, unless formation of the hairpin is prevented. Secondly, the base-pairing sequesters the RBS, specifically via a UCCCC-motif which is common to many regulatory RNAs, and prevents translation initiation. Thus there is a competition between ribosome binding and formation of the hairpin, presumably resulting in highly efficient repression of IcaR expression and tight control over this key virulence factor [28]. It is possible that many more examples of regulatory $3^{\prime}$-UTRs can be found in $S$. aureus. It is therefore encouraging that a recently developed method, named Term-seq, is capable of transcriptome-wide mapping of the 3'-ends with single-nucleotide precision, which should reveal both potentially regulatory 3'-UTRs and riboswitch activity in S. aureus [31]. 


\section{3. Regulating virulence with small RNAs}

2 Virulence in $S$. aureus is tightly linked to quorum sensing, which allows the bacterium to shift 3 between a sedentary life in a biofilm and dispersal throughout the host organism. The 4 quorum sensing itself is mediated by the export of a small peptide that is detected by a two5 component system, all encoded in the agr operon. However, the intra-cellular effector 6 molecule induced by the two-component system is RNAIII, a multifunctional small RNA, 7 which can post-transcriptionally repress certain genes and induce translation of others [1].

\section{$8 \quad 3.1$ RNAIII and virulence}

9 RNAIII has 14 stem-loops, providing a range of platforms for hybridising to different RNAs with varying affinities. Three of the loops (loops 7,13 and 14) are rich in cytosine, which enable them to anneal with the ribosome binding sites of many mRNAs (the consensus Shine-Dalgarno sequence in S. aureus is AGGAGG)[32]. Boisset and coworkers used an in silico approach to find potential targets for stem-loop 13, by computationally fusing the hypothetical 5' UTR of all open reading frames (ORFs) to the stem-loop 13 sequence, then applying an RNA folding algorithm to determine whether strong secondary structure could be formed by each of these in silico-constructed RNAs. The strongest predicted interaction was with the SA1000 mRNA, which encodes a cell-surface-anchored fibrinogen-binding protein [33]; the predicted structure would lead to an unfolding of stem-loop 13 and anti-sense binding to the whole ribosome binding region of the SA1000 mRNA. The predicted free energy of this binding is as high as $34.5 \mathrm{kcal} / \mathrm{mol}$, even though two small bulges disrupt an otherwise perfect complementarity [32]. In order to study interaction between SA1000 mRNA and RNAIII, the in vivo 5'-end of the SA1000 mRNA was determined (using primer extension), to ensure that the 5'-UTR did indeed contain the sequence needed to form the predicted structure. The structure of the SA1000 mRNA (both bound and unbound) was probed using a range of in vivo and in vitro methods, and it was found not only that the strong binding by RNAIII completely prevents the ribosome from initiating translation, but that the resulting $\sim 40$ bp double-stranded RNA also serves as cleavage site for the doublestranded endoribonuclease RNase III (see section 4 below), ensuring that the mRNA will not be translated once bound by RNAIII [32].

The many loops in RNAIII also allow more complex interactions, involving more than one region of the target $m R N A$, thus permitting sophisticated fine-tuning of binding affinities. The coa mRNA, encoding the blood-clot-promoting protein staphylocoagulase, is bound by loop 13 around the RBS, and by loop 7 further downstream, inside the coding region. Interestingly, while stem 13 is unfolded upon binding to form an imperfect duplex with the 
coa mRNA, stem 7 remains in hairpin form, so that only the loop itself base-pairs with the loop of a hairpin on the mRNA. In vitro binding assays with RNAlll deleted for either of the stem-loop 7 and 13 revealed that the primary binding event appears to be stem-loop 13 with the RBS, since binding of loop 7 to the downstream site is diminished if stem-loop 13 is removed from RNAIII [34].

Further complexity is added by the ability of RNAlll to function as an activator on certain of the bound mRNAs, in addition to its mRNA repression role. One example is hla mRNA, encoding the cytotoxic hemolysin alpha protein, which forms an intra-molecular secondary structure that sequesters the Shine-Dalgarno sequence [35]. Activation by RNAIll is thought to be achieved by formation of a duplex between the 5'-end of RNAlll and an RNA region on the hla mRNA which is complementary to the hla Shine-Dalgarno sequence. This duplex, which involves stem-loops 1, 2 and 3 of RNAIII, would liberate the Shine-Dalgarno and thus permit translation initiation [35].

SA1000, coa and hla are only three examples of many RNAlll targets (either predicted or experimentally verified). However, the amount of RNAIll is not infinite in the cell, especially in mid-exponential growth phase, where the quorum sensing system has not yet been fully activated (Figure 2). This means that the various target mRNAs must compete for RNAlll binding, especially since many of them, for example SA1000 and coa mRNAs, use the same binding site(s) on RNAIII. How this competition affects RNAlll-mediated virulence regulation in vivo remains an open question, whose resolution will require detailed studies of the in vivo turnover and localisation of the molecules involved.

Finally, it is important to note that no protein factor(s) have been identified as necessary for RNAIII interactions with SA1000 and coa mRNAs (no data is available for hla mRNA), in contrast to $E$. coli where Hfq protein is needed to promote many of the inter-molecular RNARNA regulatory events. S. aureus does encode an ortholog of $\mathrm{Hfq}$, and although several observations that $h f q$ deletion has no impact on RNA-RNA interactions have been reported $[32,34,36]$, it has been suggested that this may be highly strain-dependent [37], raising again the question of how the equilibrium of RNA-RNA interactions is handled in strains where Hfq has no role?

\subsection{Other small regulatory RNAs in $S$. aureus}

The importance of RNAIll as a central virulence regulator has led several groups to search for other regulatory RNAs in S. aureus, and more than 500 "non-coding" RNAs have now been identified [38], most of them using various forms of RNA sequencing methods [39-43]. 
1 However, the analysis methods used do not always distinguish between a true small RNA 2 (meaning a separate short transcript) and an unexpectedly long UTR. Further confounding 3 the matter is the possibility that small RNAs can be shortened by RNases and so change their regulatory specificity, and that some small RNAs are the result of processing of a longer mRNA. An attempt to re-find many of these non-coding RNAs using more modern sequencing methods and stricter inclusion criteria, succeeded in re-finding only 159 small RNAs, which nevertheless remains an impressively high number [38].

8 That said, the mere existence of a short RNA molecule does not mean that it has a regulatory function, and does not by itself identify its RNA targets. MAPS (MS2 affinity purification coupled to RNA sequencing) was developed to identify targets of a specific small RNA, with no prior knowledge of its "targetome" [44]. The MAPS technique was adapted for S. aureus, and used to identify RNA partners of the small RNA RsaA. Briefly, the 5'-end of RsaA was fused to a sequence that folds into two hairpins recognised by the MS2 coat protein. This permits the specific purification of the tagged RsaA, as well as any RNA molecules that co-purify with it. Deep sequencing of the total purified RNA (compared to a control sample without tagged RsaA) revealed potential partners, with the previously identified target mgrA mRNA at the top of the list [45,46]. Further experiments confirmed the interaction between RsaA and several mRNAs, and demonstrated that RsaA is capable of sequestering ribosome binding sites to prevent translation initiation [45].

A complementary strategy for demonstrating regulatory $S$. aureus RNA-RNA interactions in vivo, consists of co-expressing the small RNA together with the potential mRNA target with a translationally fused green fluorescent protein (GFP). The amount of GFP produced can then be quantified, either on agar plates or with a micro-plate reader, and if GFP expression is dependent on the presence of the small RNA, a direct interaction is a strong possibility [47]. While this strategy is not a global survey method, it is designed to enable rapid screening of many targets, since the small RNA and the target RNAs are expressed from separate vectors which can be combined in various ways. This strategy was initially tested with RNAIII and known interacting mRNAs, and was demonstrated to be useful for showing not only repression by small RNAs, but also RNAlll-dependent positive translational regulation of MapW (an extracellular adherence protein) $[47,48]$. The technique was further used to discover regulation by the prophage-encoded sRNA SprX2, which down-regulates the expression of extracellular complement-binding protein Ecb. It is intriguing that this protein is also down-regulated by RNAIII via a different interaction [47], and that RNAIII appears also to base-pair directly with SprX1, which is almost identical in sequence to SprX2, suggesting a complex web of interactions between small RNAs themselves as well as with mRNAs [49]. 


\section{4. RNase III}

2 Intramolecular secondary structures in an mRNA, and hybridisation with regulatory sRNAs, 3 can regulate protein expression in several ways. The ribosome binding site (RBS) can be 4 sequestered or released, sometimes upon intervention of a metabolite, but the secondary 5 structures can also serve as targets for RNase III, which specifically cleaves double6 stranded RNA. This frequently leads to a rapid degradation of any RNA with a long double7 stranded region, permitting small RNAs to initiate decay of mRNAs, provided the two RNAs 8 share sufficient base-pair complementarity [42]. Interestingly, the sites of RNase III cleavage 9 are not always randomly distributed along a double-stranded RNA, but can be directed to cleave at defined sites by base-mismatch bulges in the duplex $[42,50]$.

\subsection{RNase III acts on a multitude of targets}

An early transcriptome-wide survey of RNase III-associated RNAs was performed by deep sequencing the RNA that co-immunoprecipitated with either the wild-type RNase III (able to bind and cleave the RNA) or active site mutants of RNase III (able only to bind the RNA) [42]. This revealed a number of virulence transcripts that encode either virulence factors or regulators involved in virulence. In addition, many small regulatory RNAs were identified as likely substrates of RNase III [42]. This powerful global approach permitted the identification of potential regions of RNase III cleavage, and additional primer extension and RACE experiments were performed on many RNAs to both confirm cleavage and map the cleavage sites [42].

\subsection{RNase III removes the products of leaky and anti-sense} transcription

Tight control over transcription is a challenge for any cell, especially for organisms like $S$. aureus, where the high $\mathrm{A}+\mathrm{T}$ content in the genome (67\%) generates a multitude of sites that fortuitously resemble the $A+T$ rich consensus binding sequence for the house-keeping sigma-factor (TTGACA - 17 bp - TATAAT) [18]. Such "stochastic" transcription start sites would be expected to be equally abundant on either DNA strand, and it was therefore curious that very little anti-sense RNA was detected with strand specific RNA sequencing of a wild-type $S$. aureus. However, when an identical RNA sequencing was performed on an RNase III mutant, it was possible to detect large amounts of anti-sense RNA in the sample, 
1 indicating that RNase III initiates degradation of the RNA-RNA hybrids between RNA

2 transcribed from the two DNA strands [41]. Such a process can only remove a small fraction

3 of a highly expressed sense RNA (corresponding to a stoichiometric amount of antisense

$4 \quad$ RNA), but will be able to remove trace amounts of transcript generated by a leaky repressed

5 promoter. RNase III is therefore both a vital factor for specific post-transcriptional regulation

6 of transcripts that form duplexes with small RNAs, as well as ensuring the general fidelity of

7 the pre-transcriptional regulation.

\section{5. RNase $Y$}

10

As seen above for RNase III, many cellular processes have tightly controlled gene expression that is achieved by a precise and specific initiation of RNA decay. However, not all regulatory cleavage events take place on double stranded RNA. A genome-wide survey of $S$. aureus mutants whose disrupted genes had unknown functions but were conserved in other Gram-positive pathogens, identified a gene (dubbed conserved virulence factor $A$, $c v f A$ ) required for full haemolysin production on blood-agar plates and virulence of $S$. aureus in silkworms [51]. It was later discovered that $c v f A$ encodes RNase $\mathrm{Y}$, a protein with singlestranded endoribonucleolytic activity (independently discovered in Bacillus subtilis [52]), and its importance for virulence was further demonstrated with a murine $S$. aureus bacteraemia model [12].

\subsection{Localization of RNase $Y$}

The RNA polymerase holoenzyme is highly conserved among bacteria, but the RNA degradation machinery does not share this conservation. In $E$. coli, the membraneassociated RNase $\mathrm{E}$ is the central component of the degradosome, the major RNA degradation complex [53], but RNase $E$ is absent from many bacterial genomes, including $S$. aureus. The discovery of RNase $Y$, a membrane-associated endoribonuclease of firmicutes that interacts with other participants in RNA degradation, led to the hypothesis that RNase $Y$ plays a role similar to that of RNase E in Escherichia coli by acting as a scaffold for the formation of a degradosome-like structure [52]. The RNase Ys of B. subtilis and S. aureus were indeed shown, using bacterial-two-hybrid assay and by tandem affinity purification, to interact directly with the glycolytic enzyme enolase and the DEAD-box RNA helicase CshA $[54,55]$ The latter protein has been proposed to enable the RNA decay machinery to gain access to cleavage sites embedded in secondary structures $[52,56]$. 
1 Deletion of the $\operatorname{csh} A$ gene in $S$. aureus leads to a very poor growth of the mutant at low 2 temperatures and a defective decay of 113 RNAs. Among these is the quorum sensing 3 mRNA agr, whose consequent accumulation is presumed to cause the over-production of 4 haemolysins observed in this mutant [55,57]. Surprisingly, removal of the RNase $Y$ membrane anchor results in almost complete reversal of the $\Delta c s h A$-phenotypes.

6 Furthermore this suppressive effect is directly linked to the sub-cellular localization of RNase

$7 \mathrm{Y}$, since the re-anchoring of the anchorless RNase $\mathrm{Y}$ (via dimerisation with an enzymaticaly 8 dead RNase $\mathrm{Y}$ ) virtually eliminates the rescue of $\Delta c s h A$ phenotypes [11]. This phenomenon could be explained by access of an anchor-less RNase $Y$ to RNA molecules that are normally inaccessible when RNase $Y$ is confined to the membrane. This is consistent with preliminary results from our lab that indicate a general shortening of RNA half-lives in the anchorless RNase $Y$ mutant and offer a possible explanation of its suppressive effect on the cshA deletion. The sub-cellular localization of RNase $Y$ thus seems to play a significant role in limiting its activity. This raises the question of the localization of RNA molecules inside the cell: is there a sub-population of RNAs localized near the membrane and if so, how is this partition regulated?

Proteins destined for export or bound to the membrane frequently carry an $\mathrm{N}$-terminal signal peptide. This peptide is then recognized by the signal recognition particle, which transports the nascent protein, the translating ribosome and the mRNA being translated to the membrane [58]. This active system ensures that exported and membrane bound proteins are correctly addressed to the membrane but as a consequence also transports mRNAs that could be cleaved by the membrane-anchored RNase Y. However, combining data on RNase Y-dependent RNA decay with whose generated by a trans-membrane and signal-peptide recognition algorithm showed that for degradation of most membrane-protein-encoding mRNAs, RNase Y-activity is not rate limiting step [59]. It is therefore unlikely that the signal peptide is the major determining factor in RNase $\mathrm{Y}$-dependent mRNA degradation, and the true reason for the membrane localisation of RNase $\mathrm{Y}$ is to be sought elsewhere.

\subsection{RNase Y post-transcriptionally mediates virulence factors}

Recent experiments have shown that in an RNase $\mathrm{Y}$ deletion mutant, many virulence-related ORFs had a longer half-life and a correspondingly increased steady state abundance, although the set of virulence factors affected changes according to the experimental setup and the lineage of $S$. aureus used. This is especially evident for decay of the agr quorumsensing transcript, which is affected differently in three separate studies of RNase $Y$ mutants $[11,12,51]$. One of the most thoroughly studied examples of the effect of RNA cleavage on 
1 shown to be involved in the regulation of haemolysins, nucleases and coagulase when 2 exposed to $\alpha$-defensins or $\mathrm{H}_{2} \mathrm{O}_{2}$, which are both produced upon phagocytosis [60]. SaeS acts as a kinase sensor and SaeR as a transcription regulator, forming a two-component system. SaeP and SaeQ have been proposed to assist the Sae system in returning to its pre-activation state by negative feedback [61]. The processing of this operon by RNase $Y$ allows differential expression of the co-transcribed genes, due to a precise RNase $Y$ cleavage between saeP and saeQ (Figure 3). The cleavage induced by the RNase induces rapid degradation of the upstream RNA fragment and stabilization of the downstream fragment, leading to the prevalence of saeRS [13]. Thus, RNA degradation can enable a rapid change in expression profiles making $\mathrm{RNase} Y$ an important player in this virulenceregulation network.

A better understanding of how RNase $Y$ selects its target would certainly provide more information on its regulatory role in virulence. It is striking that the half-lives of only about a hundred open reading frames are significantly prolonged in an RNase $Y$ mutant, indicating high selectivity [11]. To show whether those RNAs are in fact cleaved by RNase $Y$ and where, Exact Mapping Of Transcriptome Ends (EMOTE) was used. EMOTE is a transcriptome-wide approach based on the ligation of a synthetic RNA oligonucleotide to the 5 ' ends of total RNA, similar to the transcription start site mapping described in section 2 (see also Figure 1). However, no pretreatment of the RNA is needed, since RNase $Y$ cleavages leave a 5' mono-phosphate ready for ligation. Comparing EMOTE data on WT and an RNase $Y$ mutant to identify 5'-ends absent from the RNase $Y$ mutant resulted in a list of 99 RNase $Y$ cleavage sites. Bioinformatic analysis of those sites showed that RNase $Y$ preferentially cleaves after a $G$ in an $A / U$-rich region but that neither condition is an absolute requirement [11]. Furthemore, deletion of 13 nucleotides surrounding the cleavage site of the sae operon did not prevent it's cleavage by RNase $\mathrm{Y}$, but rather moved the cleavage site exactly 13 nucleotides upstream, leading to the proposal that RNase $Y$ recognizes nearby secondary structures downstream of the cleavage site [13]. Although bioinformatic analyses of the EMOTE data did not reveal a common secondary structure within 50 nucleotides of the RNase $Y$ cleavage sites, Marincola and coworkers identified a sequence element named Rrs (for RNase $Y$ recognition sequence) immediately downstream of the cleavage site which can form a putative secondary structure with its counterpart, located a further $\sim 80$ nucleotides downstream. RNase $Y$ cleavage was observed whenever the $\mathrm{Rrs}$ and its counterpart were present, but none when they were absent, strongly indicating that Rrs and its counterpart forms a structure that guides RNase $Y$ to correctly cleave the saePQRS transcript [13]. 


\section{6. Conclusion}

2 It is not known how many processes are regulated post-transcriptionally by RNA-RNA

3 interactions in $S$. aureus, but new small RNAs and new interactions, demonstrated or 4 potential, are published almost monthly: it appears that virtually every major metabolic or

5 virulence pathway has a small RNA or a regulatory RNA structure somewhere. Is there

6 something about $S$. aureus that makes post-transcriptional regulation preferable? The small

7 number of sigma factors in $S$. aureus must certainly play a role in this, but it is unclear

8 whether the scarcity of sigma factors caused the efficient RNA-mediated regulation to

9 evolve, or whether the efficient RNA-mediated regulation made additional sigma factors

10 redundant. Moreover, commensal bacteria like $S$. aureus, which are adapted to living within

11 the human nasopharynx, experience a changing environment with respects to the presence

12 of other commensals, mucus, host immune system, physical stress etc., but a relatively

13 constant temperature. The formation of RNA-RNA duplexes is sensitive to temperature,

14 especially if they are short, as in the case of the initial interactions between the hairpin-loops

15 of RNAIII and their targets, and a constant temperature makes duplex formation predictable

16 and tractable for fine-tuning - a requirement for precise regulation. Interestingly, many

17 mutants in the RNA decay machinery of $S$. aureus grow very poorly (or not at all) when

18 exposed to temperature changes of as little as 5 to $7^{\circ} \mathrm{C}$ (i.e at $42^{\circ} \mathrm{C}$ or $30^{\circ} \mathrm{C}$ ) $[57,62,63$ ), 19 perhaps due to misfolding of RNAs. Furthermore, both RNase III and RNase $Y$ appear to 20 base their high cleavage specificity on nearby secondary structure elements [13,42], which 21 suggests that the equilibrium between the three states: structured, single-stranded and 22 actively translated is vital for determining the fate of an RNA molecule. A possible way to study such processes in a transcriptome-wide manner would be to re-analyse the 99 known

24 RNase $\mathrm{Y}$ cleavage sites [11] using the knowledge gained from the detailed analyses of the 25 cleavage site in the sae transcript [13]. Ideally such an in silico analysis could be enhanced 26 immensely both by transcriptome-wide probing of RNA secondary structures, several 27 protocols for which have now been published [64], and by intra-cellular localisation of RNase $28 \mathrm{Y}$ and its RNA targets.

30 Acknowledgements:

31 We would like to thank Dr. Dave Lane for reading, commenting on and correcting the English 32 in the manuscript.

34 Funding: 
1 This work was supported by the Paul Sabatier University, Toulouse and the Centre for 2 Integrative Biology.

\section{Conflict of interest:}

5 The authors have nothing to disclose.

6

7

\section{Figure legends:}

Figure 1, Preparing RNA for 5'-end sequencing.

RNA is shown in green and DNA in light blue. Known sequence of synthetic oligos is shown as bold lines and unknown sequence as fine lines. A) Four major types of 5 '-end has been found in $S$. aureus: original transcripts with tri-phosphorylated 5'-ends, monophosphorylated transcripts produced by a pyrophosphohydrolase such as $\mathrm{RppH}$, monophosphorylated down-stream products produced by RNases such as RNase Y or RNase III, and non-phosphorylated down-stream products formed by toxin RNase-mediated cleavage or by autocatalyzed. Note that di-phosphorylated and NAD-capped RNA species might also exist, but have yet to be identified. B) To identify the first nucleotide of the 5'-end in monophosphorylated RNA, an oligo with known sequence is ligated to all the 5'-ends in the sample of cellular RNA. The RNA is then reverse transcribed to CDNA (for example using a random primer), which will now have the reverse complement of the known oligo sequence at its 3'-end. A primer that hybridises to this known sequence can then be used to perform a sequencing reaction using massive parallel sequencing methods. C) Since monophosphorylated RNA species can be readily ligated to the synthetic oligo, it is sometimes convenient to remove all or most of the mono-phosphorylated RNA in the sample when the object of interest is tri-phosphorylated or non-phosphorylated species. Mono-phosphorylated RNA can easily be removed by 5 '-exonucleases that are specific for mono-phosphorylated RNA, such as Terminator or XRN1. D) Non-phosphorylated 5'-ends cannot act as substrates for a ligation, and must be mono-phosphorylated by polynucleotide kinase before they can be ligated to a synthetic oligo (see B). E) Tri-phosphorylated 5'-ends cannot act as substrates for a ligation, and must be converted to mono-phosphates by a pyrophosphatase (e.g. Tobacco Acid Pyrophosphatase or $\mathrm{RppH}$ ) before they can be ligated to a synthetic oligo (see B). F and G) The sequencing reads will consist of a short region of known sequence (shown in bold uppercase and corresponding to a small part of the original oligo that was ligated to the RNA) followed immediately by the first nucleotide of the original RNA 5 '-end (shown in lowercase). The oligo sequence (bold uppercase) can be removed in silico 
1 from each read, whereupon the sequence original 5'-end of the RNA can be mapped into the

2 genome of the examined organism to identify the exact transcription start site.

4 Figure 2, Examples of virulence factors regulated by RNAIII.

5 RNAIII is regulated positively by the quorum sensing agr operon. Post-transcriptional regulation is represented by coloured lines, with up-regulation indicated by green arrows and down-regulation by red cross-bars. RNAIII in turn positively regulates MapW and Hla at a post transcriptional level. MapW and Hla prevents leucocyte adhesion and promotes dissemination by lysing host cells, respectively. RNAllI also negatively regulates LytM, Rot, Spa and SarT which respectively promotes: autolysis (via LytM), blood cell toxicity (via Rot) and expression of an IgG binding protein (via SarT). The schematic is simplified for clarity, and the mediators presented have been reported to have many other functions however only the interactions discussed in this review have been included. For a more complete interaction map, please see [3].

Figure 3, RNase $\mathrm{Y}$ cleavage of the sae operon maintains correct regulation.

The saePQRS operon is represented schematically. The RNase $\mathrm{Y}$ cleavage site is shown alongside the Rrs counterpart identified by Marincola and coworkers [13]. (A) Processing of the operon by RNase $Y$ leads to rapid degradation of the upstream fragment which in turn leads to the prevalence of SaeRS inside the cell. Phosphorylation of the transcriptional regulator SaeR leads to expression of virulence factors and SaePQ. (B) Absence of RNase $Y$ cleavage results in higher expression of saeP which applies a negative feedback on SaeRS, presumably causing loss of SaeRS function.

\section{References}

[1] R.P. Novick, H.F. Ross, S.J. Projan, J. Kornblum, B. Kreiswirth, S. Moghazeh, Synthesis of staphylococcal virulence factors is controlled by a regulatory RNA molecule, EMBO J. 12 (1993) 3967-3975.

[2] B. Felden, P. Bouloc, Regulatory RNAs in bacteria: From identification to function, Methods San Diego Calif. 117 (2017) 1-2. doi:10.1016/j.ymeth.2017.03.018.

[3] P. Fechter, I. Caldelari, E. Lioliou, P. Romby, Novel aspects of RNA regulation in Staphylococcus aureus, FEBS Lett. 588 (2014) 2523-2529. doi:10.1016/j.febslet.2014.05.037.

[4] E.G.H. Wagner, P. Romby, Small RNAs in bacteria and archaea: who they are, what they do, and how they do it, Adv. Genet. 90 (2015) 133-208. doi:10.1016/bs.adgen.2015.05.001.

[5] D. Bronesky, Z. Wu, S. Marzi, P. Walter, T. Geissmann, K. Moreau, F. Vandenesch, I. Caldelari, P. Romby, Staphylococcus aureus RNAIII and Its Regulon Link Quorum Sensing, Stress Responses, Metabolic Adaptation, and Regulation of Virulence Gene 
Expression, Annu. Rev. Microbiol. 70 (2016) 299-316. doi:10.1146/annurev-micro102215-095708.

[6] S. Durand, A. Tomasini, F. Braun, C. Condon, P. Romby, sRNA and mRNA turnover in Gram-positive bacteria, FEMS Microbiol. Rev. 39 (2015) 316-330.

doi:10.1093/femsre/fuv007.

[7] H.F.L. Wertheim, D.C. Melles, M.C. Vos, W. van Leeuwen, A. van Belkum, H.A. Verbrugh, J.L. Nouwen, The role of nasal carriage in Staphylococcus aureus infections, Lancet Infect. Dis. 5 (2005) 751-762. doi:10.1016/S1473-3099(05)70295-4.

[8] K. Morikawa, Y. Inose, H. Okamura, A. Maruyama, H. Hayashi, K. Takeyasu, T. Ohta, A new staphylococcal sigma factor in the conserved gene cassette: functional significance and implication for the evolutionary processes, Genes Cells Devoted Mol. Cell. Mech. 8 (2003) 699-712.

[9] L.N. Shaw, C. Lindholm, T.K. Prajsnar, H.K. Miller, M.C. Brown, E. Golonka, G.C. Stewart, A. Tarkowski, J. Potempa, Identification and characterization of sigma, a novel component of the Staphylococcus aureus stress and virulence responses, PloS One. 3 (2008) e3844. doi:10.1371/journal.pone.0003844.

[10] K.L. Anderson, C.M. Roux, M.W. Olson, T.T. Luong, C.Y. Lee, R. Olson, P.M. Dunman, Characterizing the effects of inorganic acid and alkaline shock on the Staphylococcus aureus transcriptome and messenger RNA turnover, FEMS Immunol. Med. Microbiol. 60 (2010) 208-250. doi:10.1111/j.1574-695X.2010.00736.x.

[11] V. Khemici, J. Prados, P. Linder, P. Redder, Decay-Initiating Endoribonucleolytic Cleavage by RNase $Y$ Is Kept under Tight Control via Sequence Preference and Subcellular Localisation, PLoS Genet. 11 (2015) e1005577. doi:10.1371/journal.pgen.1005577.

[12] G. Marincola, T. Schäfer, J. Behler, J. Bernhardt, K. Ohlsen, C. Goerke, C. Wolz, RNase $Y$ of Staphylococcus aureus and its role in the activation of virulence genes, Mol. Microbiol. 85 (2012) 817-832. doi:10.1111/j.1365-2958.2012.08144.x.

[13] G. Marincola, C. Wolz, Downstream element determines RNase Y cleavage of the saePQRS operon in Staphylococcus aureus, Nucleic Acids Res. (2017). doi:10.1093/nar/gkx296.

[14] J.M. Schifano, I.O. Vvedenskaya, J.G. Knoblauch, M. Ouyang, B.E. Nickels, N.A. Woychik, An RNA-seq method for defining endoribonuclease cleavage specificity identifies dual rRNA substrates for toxin MazF-mt3, Nat. Commun. 5 (2014) 3538. doi:10.1038/ncomms4538.

[15] C.M. Sharma, J. Vogel, Differential RNA-seq: the approach behind and the biological insight gained, Curr. Opin. Microbiol. 19 (2014) 97-105. doi:10.1016/j.mib.2014.06.010.

[16] V. Pelechano, W. Wei, P. Jakob, L.M. Steinmetz, Genome-wide identification of transcript start and end sites by transcript isoform sequencing, Nat. Protoc. 9 (2014) 1740-1759. doi:10.1038/nprot.2014.121.

[17] P. Redder, Using EMOTE to map the exact 5'-ends of processed RNA on a transcriptome-wide scale, Methods Mol. Biol. Clifton NJ. 1259 (2015) 69-85. doi:10.1007/978-1-4939-2214-7_5.

[18] J. Prados, P. Linder, P. Redder, TSS-EMOTE, a refined protocol for a more complete and less biased global mapping of transcription start sites in bacterial pathogens, BMC Genomics. 17 (2016) 849. doi:10.1186/s12864-016-3211-3.

[19] C.L. Kirkpatrick, D. Martins, P. Redder, A. Frandi, J. Mignolet, J.B. Chapalay, M. Chambon, G. Turcatti, P.H. Viollier, Growth control switch by a DNA-damage-inducible toxin-antitoxin system in Caulobacter crescentus, Nat. Microbiol. (2016) 16008.

[20] A. Fouquier d'Hérouel, F. Wessner, D. Halpern, J. Ly-Vu, S.P. Kennedy, P. Serror, E. Aurell, F. Repoila, A simple and efficient method to search for selected primary transcripts: non-coding and antisense RNAs in the human pathogen Enterococcus faecalis, Nucleic Acids Res. 39 (2011) e46. doi:10.1093/nar/gkr012.

[21] J. Vogel, V. Bartels, T.H. Tang, G. Churakov, J.G. Slagter-Jäger, A. Hüttenhofer, E.G.H. Wagner, RNomics in Escherichia coli detects new sRNA species and indicates parallel transcriptional output in bacteria, Nucleic Acids Res. 31 (2003) 6435-6443. 
[22] L. Argaman, R. Hershberg, J. Vogel, G. Bejerano, E.G. Wagner, H. Margalit, S. Altuvia, Novel small RNA-encoding genes in the intergenic regions of Escherichia coli, Curr. Biol. CB. 11 (2001) 941-950.

[23] B.A. Bensing, B.J. Meyer, G.M. Dunny, Sensitive detection of bacterial transcription initiation sites and differentiation from RNA processing sites in the pheromone-induced plasmid transfer system of Enterococcus faecalis, Proc. Natl. Acad. Sci. U. S. A. 93 (1996) 7794-7799.

[24] C.M. Sharma, S. Hoffmann, F. Darfeuille, J. Reignier, S. Findeiss, A. Sittka, S. Chabas, K. Reiche, J. Hackermüller, R. Reinhardt, P.F. Stadler, J. Vogel, The primary transcriptome of the major human pathogen Helicobacter pylori, Nature. 464 (2010) 250-255. doi:10.1038/nature08756.

[25] F. Amman, M.T. Wolfinger, R. Lorenz, I.L. Hofacker, P.F. Stadler, S. Findeiß, TSSAR: TSS annotation regime for dRNA-seq data, BMC Bioinformatics. 15 (2014) 89. doi:10.1186/1471-2105-15-89.

[26] H. Jorjani, M. Zavolan, TSSer: an automated method to identify transcription start sites in prokaryotic genomes from differential RNA sequencing data, Bioinforma. Oxf. Engl. 30 (2014) 971-974. doi:10.1093/bioinformatics/btt752.

[27] G. Dugar, A. Herbig, K.U. Förstner, N. Heidrich, R. Reinhardt, K. Nieselt, C.M. Sharma, High-resolution transcriptome maps reveal strain-specific regulatory features of multiple Campylobacter jejuni isolates, PLoS Genet. 9 (2013) e1003495. doi:10.1371/journal.pgen.1003495.

[28] I. Ruiz de los Mozos, M. Vergara-Irigaray, V. Segura, M. Villanueva, N. Bitarte, M. Saramago, S. Domingues, C.M. Arraiano, P. Fechter, P. Romby, J. Valle, C. Solano, I. Lasa, A. Toledo-Arana, Base pairing interaction between 5'- and 3'-UTRs controls icaR mRNA translation in Staphylococcus aureus, PLoS Genet. 9 (2013) e1004001. doi:10.1371/journal.pgen.1004001.

[29] U. Mäder, P. Nicolas, M. Depke, J. Pané-Farré, M. Debarbouille, M.M. van der KooiPol, C. Guérin, S. Dérozier, A. Hiron, H. Jarmer, A. Leduc, S. Michalik, E. Reilman, M. Schaffer, F. Schmidt, P. Bessières, P. Noirot, M. Hecker, T. Msadek, U. Völker, J.M. van Dijl, Staphylococcus aureus Transcriptome Architecture: From Laboratory to Infection-Mimicking Conditions, PLoS Genet. 12 (2016) e1005962. doi:10.1371/journal.pgen.1005962.

[30] V. Khemici, A.J. Carpousis, The RNA degradosome and poly (A) polymerase of Escherichia coli are required in vivo for the degradation of small mRNA decay intermediates containing REP-stabilizers, Mol. Microbiol. 51 (2004) 777-790.

[31] D. Dar, M. Shamir, J.R. Mellin, M. Koutero, N. Stern-Ginossar, P. Cossart, R. Sorek, Term-seq reveals abundant ribo-regulation of antibiotics resistance in bacteria, Science. 352 (2016) aad9822. doi:10.1126/science.aad9822.

[32] S. Boisset, T. Geissmann, E. Huntzinger, P. Fechter, N. Bendridi, M. Possedko, C. Chevalier, A.C. Helfer, Y. Benito, A. Jacquier, C. Gaspin, F. Vandenesch, P. Romby, Staphylococcus aureus RNAIII coordinately represses the synthesis of virulence factors and the transcription regulator Rot by an antisense mechanism, Genes Dev. 21 (2007) 1353-1366. doi:10.1101/gad.423507.

[33] X. Liang, C. Yu, J. Sun, H. Liu, C. Landwehr, D. Holmes, Y. Ji, Inactivation of a twocomponent signal transduction system, SaeRS, eliminates adherence and attenuates virulence of Staphylococcus aureus, Infect. Immun. 74 (2006) 4655-4665. doi:10.1128/IAI.00322-06.

[34] C. Chevalier, S. Boisset, C. Romilly, B. Masquida, P. Fechter, T. Geissmann, F. Vandenesch, P. Romby, Staphylococcus aureus RNAIll binds to two distant regions of coa mRNA to arrest translation and promote mRNA degradation, PLoS Pathog. 6 (2010) e1000809. doi:10.1371/journal.ppat.1000809.

[35] E. Morfeldt, D. Taylor, A. von Gabain, S. Arvidson, Activation of alpha-toxin translation in Staphylococcus aureus by the trans-encoded antisense RNA, RNAIII, EMBO J. 14 (1995) 4569-4577. 
[36] C. Bohn, C. Rigoulay, P. Bouloc, No detectable effect of RNA-binding protein Hfq absence in Staphylococcus aureus, BMC Microbiol. 7 (2007) 10. doi:10.1186/14712180-7-10.

[37] Y. Liu, N. Wu, J. Dong, Y. Gao, X. Zhang, C. Mu, N. Shao, G. Yang, Hfq is a global regulator that controls the pathogenicity of Staphylococcus aureus, PloS One. 5 (2010). doi:10.1371/journal.pone.0013069.

[38] M. Sassi, Y. Augagneur, T. Mauro, L. Ivain, S. Chabelskaya, M. Hallier, O. Sallou, B. Felden, SRD: a Staphylococcus regulatory RNA database, RNA N. Y. N. 21 (2015) 1005-1017. doi:10.1261/rna.049346.114.

[39] M. Beaume, D. Hernandez, L. Farinelli, C. Deluen, P. Linder, C. Gaspin, P. Romby, J. Schrenzel, P. Francois, Cartography of methicillin-resistant $S$. aureus transcripts: detection, orientation and temporal expression during growth phase and stress conditions, PloS One. 5 (2010) e10725. doi:10.1371/journal.pone.0010725.

[40] C. Bohn, C. Rigoulay, S. Chabelskaya, C.M. Sharma, A. Marchais, P. Skorski, E. Borezée-Durant, R. Barbet, E. Jacquet, A. Jacq, D. Gautheret, B. Felden, J. Vogel, P. Bouloc, Experimental discovery of small RNAs in Staphylococcus aureus reveals a riboregulator of central metabolism, Nucleic Acids Res. 38 (2010) 6620-6636. doi:10.1093/nar/gkq462.

[41] I. Lasa, A. Toledo-Arana, A. Dobin, M. Villanueva, I.R. de los Mozos, M. VergaraIrigaray, V. Segura, D. Fagegaltier, J.R. Penadés, J. Valle, C. Solano, T.R. Gingeras, Genome-wide antisense transcription drives mRNA processing in bacteria, Proc. Natl. Acad. Sci. U. S. A. 108 (2011) 20172-20177. doi:10.1073/pnas.1113521108.

[42] E. Lioliou, C.M. Sharma, I. Caldelari, A.-C. Helfer, P. Fechter, F. Vandenesch, J. Vogel, P. Romby, Global regulatory functions of the Staphylococcus aureus endoribonuclease III in gene expression, PLoS Genet. 8 (2012) e1002782. doi:10.1371/journal.pgen.1002782.

[43] B.P. Howden, M. Beaume, P.F. Harrison, D. Hernandez, J. Schrenzel, T. Seemann, P. Francois, T.P. Stinear, Analysis of the small RNA transcriptional response in multidrugresistant Staphylococcus aureus after antimicrobial exposure, Antimicrob. Agents Chemother. 57 (2013) 3864-3874. doi:10.1128/AAC.00263-13.

[44] D. Lalaouna, E. Massé, Identification of sRNA interacting with a transcript of interest using MS2-affinity purification coupled with RNA sequencing (MAPS) technology, Genomics Data. 5 (2015) 136-138. doi:10.1016/j.gdata.2015.05.033.

[45] A. Tomasini, K. Moreau, J. Chicher, T. Geissmann, F. Vandenesch, P. Romby, S. Marzi, I. Caldelari, The RNA targetome of Staphylococcus aureus non-coding RNA RsaA: impact on cell surface properties and defense mechanisms, Nucleic Acids Res. 45 (2017) 6746-6760. doi:10.1093/nar/gkx219.

[46] C. Romilly, C. Lays, A. Tomasini, I. Caldelari, Y. Benito, P. Hammann, T. Geissmann, S. Boisset, P. Romby, F. Vandenesch, A non-coding RNA promotes bacterial persistence and decreases virulence by regulating a regulator in Staphylococcus aureus, PLoS Pathog. 10 (2014) e1003979. doi:10.1371/journal.ppat.1003979.

[47] L. Ivain, V. Bordeau, A. Eyraud, M. Hallier, S. Dreano, P. Tattevin, B. Felden, S. Chabelskaya, An in vivo reporter assay for sRNA-directed gene control in Grampositive bacteria: identifying a novel sRNA target in Staphylococcus aureus, Nucleic Acids Res. 45 (2017) 4994-5007. doi:10.1093/nar/gkx190.

[48] Y. Liu, C. Mu, X. Ying, W. Li, N. Wu, J. Dong, Y. Gao, N. Shao, M. Fan, G. Yang, RNAIII activates map expression by forming an RNA-RNA complex in Staphylococcus aureus, FEBS Lett. 585 (2011) 899-905. doi:10.1016/j.febslet.2011.02.021.

[49] M. Kathirvel, H. Buchad, M. Nair, Enhancement of the pathogenicity of Staphylococcus aureus strain Newman by a small noncoding RNA SprX1, Med. Microbiol. Immunol. (Berl.). 205 (2016) 563-574. doi:10.1007/s00430-016-0467-9.

[50] C. Romilly, C. Chevalier, S. Marzi, B. Masquida, T. Geissmann, F. Vandenesch, E. Westhof, P. Romby, Loop-loop interactions involved in antisense regulation are processed by the endoribonuclease III in Staphylococcus aureus, RNA Biol. 9 (2012) 1461-1472. doi:10.4161/rna.22710. 
[51] C. Kaito, K. Kurokawa, Y. Matsumoto, Y. Terao, S. Kawabata, S. Hamada, K. Sekimizu, Silkworm pathogenic bacteria infection model for identification of novel virulence genes, Mol. Microbiol. 56 (2005) 934-944. doi:10.1111/j.13652958.2005.04596.x.

[52] K. Shahbabian, A. Jamalli, L. Zig, H. Putzer, RNase Y, a novel endoribonuclease, initiates riboswitch turnover in Bacillus subtilis, EMBO J. 28 (2009) 3523-3533. doi:10.1038/emboj.2009.283.

[53] G.A. Mackie, RNase E: at the interface of bacterial RNA processing and decay, Nat. Rev. Microbiol. 11 (2013) 45-57. doi:10.1038/nrmicro2930.

[54] C.M. Roux, J.P. DeMuth, P.M. Dunman, Characterization of components of the Staphylococcus aureus mRNA degradosome holoenzyme-like complex, J. Bacteriol. 193 (2011) 5520-5526. doi:10.1128/JB.05485-11.

[55] C. Giraud, S. Hausmann, S. Lemeille, J. Prados, P. Redder, P. Linder, The C-terminal region of the RNA helicase CshA is required for the interaction with the degradosome and turnover of bulk RNA in the opportunistic pathogen Staphylococcus aureus, RNA Biol. 12 (2015) 658-674. doi:10.1080/15476286.2015.1035505.

[56] M. Lehnik-Habrink, H. Pförtner, L. Rempeters, N. Pietack, C. Herzberg, J. Stülke, The RNA degradosome in Bacillus subtilis: identification of CshA as the major RNA helicase in the multiprotein complex, Mol. Microbiol. 77 (2010) 958-971. doi:10.1111/j.13652958.2010.07264.x.

[57] S. Oun, P. Redder, J.-P. Didier, P. François, A.-R. Corvaglia, E. Buttazzoni, C. Giraud, M. Girard, J. Schrenzel, P. Linder, The CshA DEAD-box RNA helicase is important for quorum sensing control in Staphylococcus aureus, RNA Biol. 10 (2013) 157-165. doi:10.4161/rna.22899.

[58] M.M. Elvekrog, P. Walter, Dynamics of co-translational protein targeting, Curr. Opin. Chem. Biol. 29 (2015) 79-86. doi:10.1016/j.cbpa.2015.09.016.

[59] P. Redder, How does sub-cellular localization affect the fate of bacterial mRNA?, Curr. Genet. 62 (2016) 687-690. doi:10.1007/s00294-016-0587-1.

[60] T. Geiger, C. Goerke, M. Mainiero, D. Kraus, C. Wolz, The virulence regulator Sae of Staphylococcus aureus: promoter activities and response to phagocytosis-related signals, J. Bacteriol. 190 (2008) 3419-3428. doi:10.1128/JB.01927-07.

[61] D.-W. Jeong, H. Cho, H. Lee, C. Li, J. Garza, M. Fried, T. Bae, Identification of the P3 promoter and distinct roles of the two promoters of the SaeRS two-component system in Staphylococcus aureus, J. Bacteriol. 193 (2011) 4672-4684. doi:10.1128/JB.0035311.

[62] P. Linder, S. Lemeille, P. Redder, Transcriptome-wide analyses of 5'-ends in RNase J mutants of a gram-positive pathogen reveal a role in RNA maturation, regulation and degradation, PLoS Genet. 10 (2014) e1004207. doi:10.1371/journal.pgen.1004207.

[63] J. Armitano, P. Redder, V.A. Guimarães, P. Linder, An Essential Factor for High Mg2+ Tolerance of Staphylococcus aureus, Front. Microbiol. 7 (2016) 1888. doi:10.3389/fmicb.2016.01888.

[64] C. Del Campo, Z. Ignatova, Probing dimensionality beyond the linear sequence of mRNA, Curr. Genet. 62 (2016) 331-334. doi:10.1007/s00294-015-0551-5. 
Original transcript (unprocessed)

5' PPP

Pyrophosphate removal (e.g. $\mathrm{RppH}$ )

$5^{\prime} \mathrm{P}$

Cleaved by RNase (e.g. RNase Y)

RNA Oligo

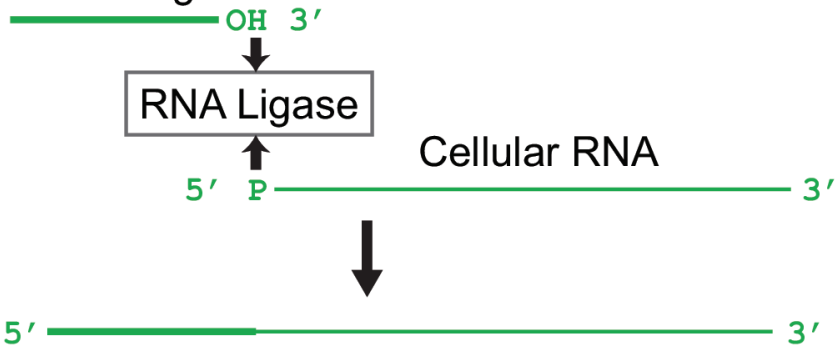

$5^{\prime} \mathrm{P}$

Cleaved by RNase (e.g. toxin)

5' $\mathrm{HO}$

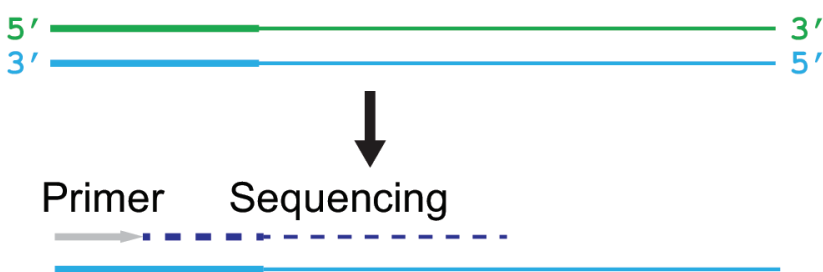

C

Mono-phosphorylated RNAs removed

with 5' mono-phosphate specific

exonuclease (ex. XRN1)

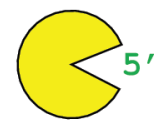

$P$

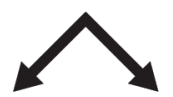

D

Non-phosporylated 5'-end

(generated by RNase-cleavage)

5' HO

Polynucleotide Kinase

5. $P$ Ready for ligation

E
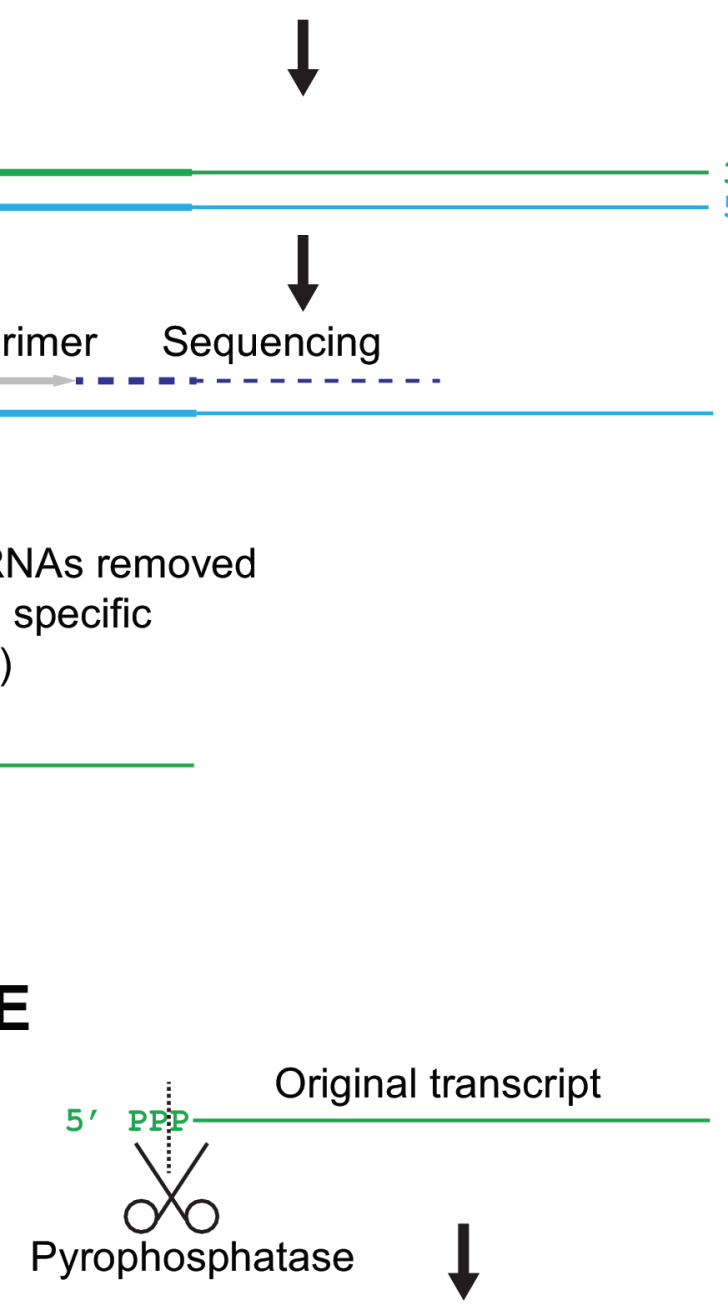

5. P_ Ready for ligation

G

Sequencing read

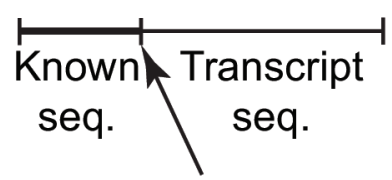

Ligation junction

Ligation junction

L_ _ _ _ _ . _ .

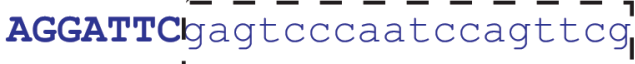
AGGATTC, I cgattcagggacacgtd AGGATTC gaaacctatatatcccagl AGGATTClactttactagtgaaaata

Sequences of 5' extremeties 


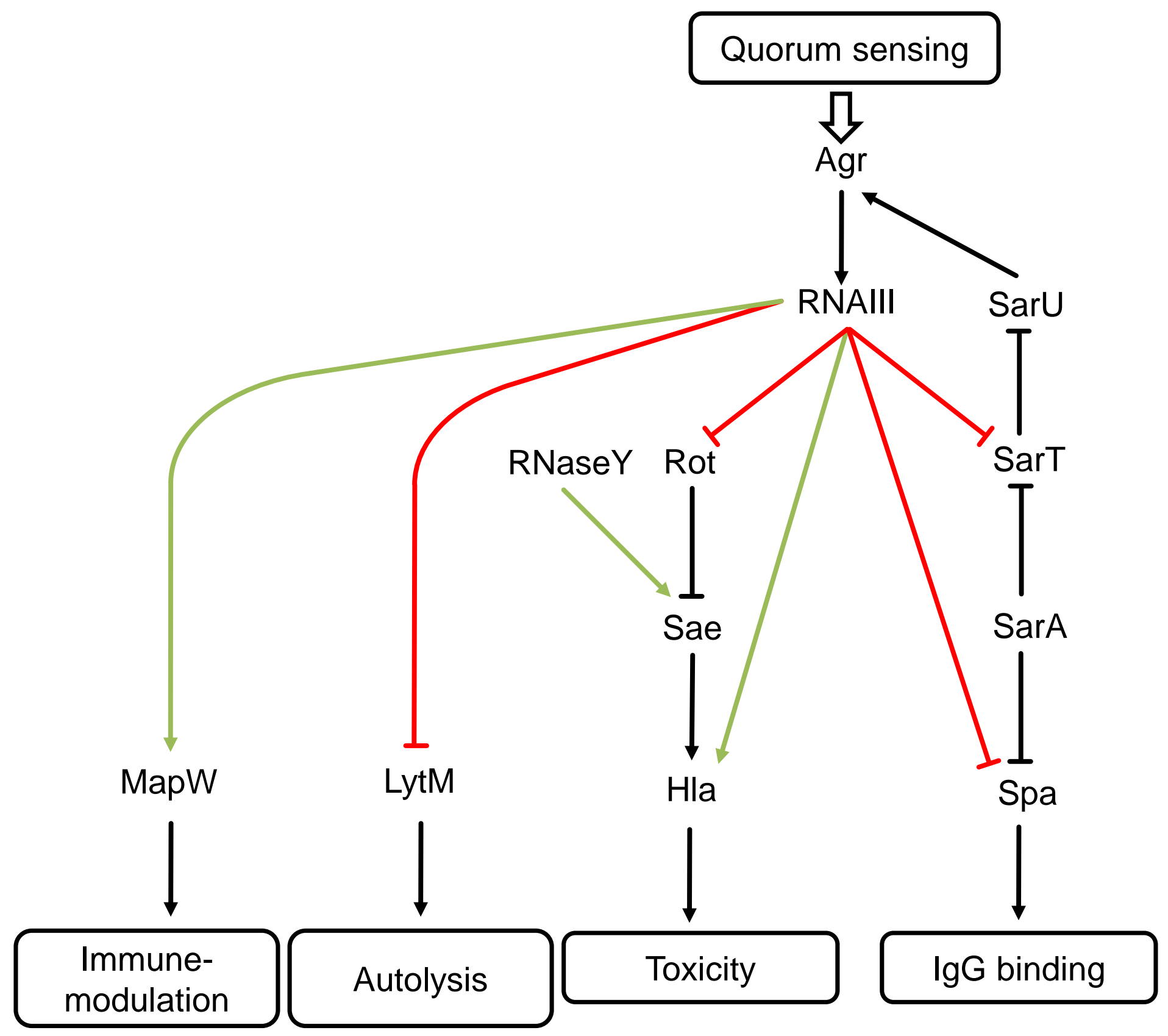




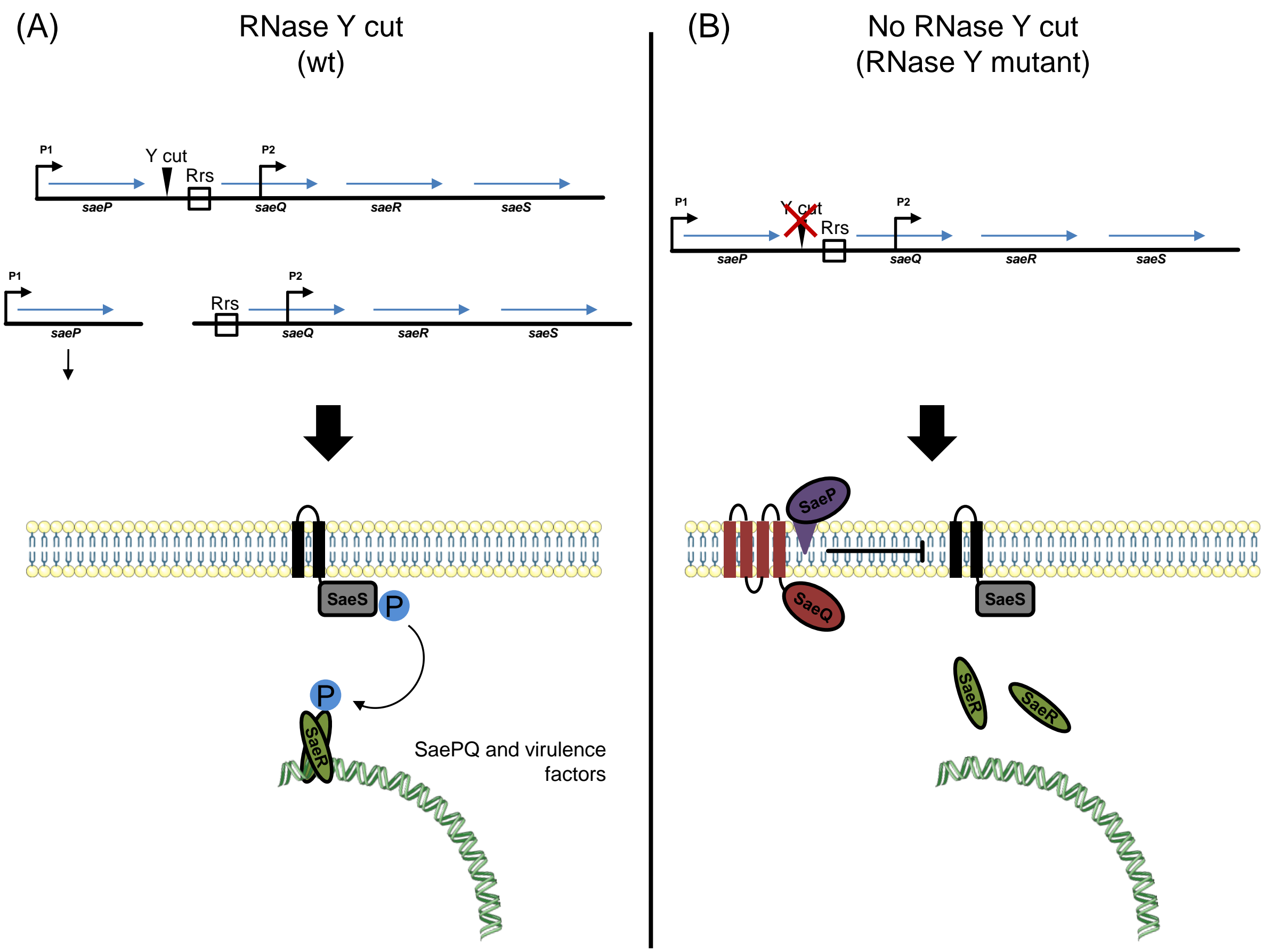

